\title{
An Exact Formulation of Coupled-Mode Theory for Coupled-Cavity Lasers
}

\author{
ROBERT J. LANG, MEMBER, IEEE, AND AMNON YARIV, FELLOW, IEEE
}

\begin{abstract}
We derive coupled-mode rate equations for coupled-cavity lasers using a new approach. The method, based on the MittagLeffler theorem, is exact. We compare the coupling coefficients to those derived by several different approximations.
\end{abstract}

\section{INTRODUCTION}

$I^{1}$ THE world of semiconductor lasers, coupled-cavity lasers are of interest for two reasons. First, intentionally coupled lasers have been demonstrated to exhibit single-longitudinal-mode oscillation under small-signal modulation, FM modulation capabilities, and bistable operation [1], [2]. Second, a coupled-cavity laser makes a good model for a laser system in which there is optical feedback. For example, if the laser output is being coupled into an optical fiber, any stray reflection back into the laser constitutes a second cavity.

The problem of analyzing the modes and/or dynamic response of a coupled-cavity laser generally boils down to developing a time-domain description of the optical field, e.g., a set of rate equations. The rate equation for the inversion, since it depends only upon the optical intensity and not the phase, is far easier to obtain. Over the years, several techniques have been developed to produce a set of optical rate equations. Generally, they are based on modal expansions in terms of either the longitudinal modes of the composite resonator (composite resonator theory) or in terms of the modes of the individual cavities (coupled-mode theory).

Composite resonator theories have the advantage of minimizing the number of variables necessary to describe the optical field. Indeed, for a coupled-cavity laser running in a single longitudinal mode, only a single variable is needed-the complex amplitude of the composite cavity mode. The simplicity inherent in such a treatment has led to relatively simple analytic expressions for the dynamic response and noise properties of coupled-cavity lasers [3]. The drawback to composite resonator theory is that one must solve the dispersion relations (and in some cases, solve for the complete fields) in steady state before a set of rate equations can be formulated.

Manuscript received February 17, 1987; revised July 30, 1987. This work was supported by the National Science Foundation and the Office of Naval Research.

R. L. Lang was with the California Institute of Technology, Pasadena, CA 91125 . He is now with the Jet Propulsion Laboratory, Pasadena, CA 91109

A. Yariv is with the California Institute of Technology, Pasadena, CA 91125

IEEE Log Number 8717454.
On the other hand, coupled-mode theory-based on an expansion of the field in eigenmodes of the individual laser cavities-increases the number of variables necessary to describe the optical field, but yields a more intuitive description of the field. If multiple longitudinal modes of the system are present, the number of variables is less attractive in a composite resonator theory (in which a new variable for each resonator mode is required). Early coupled-mode treatments [4] required lossless cavities and lossless couplings, conditions that are hardly met in current applications. More recently, Marcuse [5], [6] developed a coupled-mode description in terms of arbitrary cavities; he allowed for the selection of arbitrary boundary conditions on the fields chosen as the individual cavity modes to allow for matching the individual cavity modes to the composite cavity modes.

Unfortunately, the coefficients that appeared in the general coupled-mode description depended upon overlap integrals between the individual cavity modes and the composite cavity modes. In fact, each coupling coefficient was a sum of several such overlap integrals. Thus, it became necessary, again, to solve for the composite cavity modes in steady state to formulate a time-domain set of equations for the optical field.

It is not necessary, however, to solve for compositecavity fields to formulate a coupled-mode theory of coupled resonators. In fact, it is not even necessary to make any approximations to formulate the theory. To actually use coupled-mode theory, it is necessary to truncate the set of modes to some finite number and invoke the adiabatic approximation that the equations are valid for timevarying gain. In this paper, we derive a set of exact coupled-mode equations for a general system of two lasers; we apply the theory to the most common geometry of coupled-cavity laser, that of two axially coupled cavities, and we compare the results to those of existing theories.

\section{General Theory}

Before we proceed, one caveat is in order; all rate equation descriptions of the optical field have implicitly made an assumption that the instantaneous value of the carrier density (or equivalently, gain, or Fermi level) determines the evolution of the field. Because this approximation assumes that the optical field adiabatically follows the characteristics of the resonator, we refer to it as "the adiabatic approximation." It is definitely an approximation; the 
vector wave equation, from which all rate equations must ultimately proceed, is

$$
\nabla^{2} E(x, t)-\mu \frac{\partial^{2}}{\partial t^{2}}[E(x, t)+P(x, t)]=0
$$

where $P$ is the induced polarization and includes the effects of laser gain. Clearly, (1) and any system derived from it contain time derivatives of the gain. Those time derivatives are almost always neglected, frequently without comment.

One reason the approximation is rarely noted is that in most cases of interest (e.g., semiconductor lasers), it is a very good one. One can easily estimate the size of the incurred error [7]; typically, it scales with the ratio between the frequencies at which the gain fluctuates and the optical frequency, a ratio that is usually vanishingly small. And, of course, if the gain remains fixed, there is no approximation at all.

We point this out because our coupled mode equations, like all others, are exact only for fixed gain. By invoking the adiabatic approximation, however, they can be used for time-varying gain distributions as well. Thus, they may be used to analyze coupled-cavity laser dynamics.

We take the field to be represented by a complex scalar amplitude $F$ and the polarization to be characterized by a fixed complex dielectric constant $\epsilon_{r}$. The wave equation becomes

$$
\nabla^{2} F(x, t)-\frac{1}{c^{2}} \epsilon_{r} \frac{\partial^{2}}{\partial t^{2}} F(x, t)=0 .
$$

We choose the time factor $\exp (j \omega t)$ and Fourier transform equation (2). An operator $\partial / \partial t$ becomes an algebraic multiplier $j \omega$, and transformed variables are denoted by a tilde. The wave equation becomes a Helmholtz equation,

$$
\nabla^{2} \tilde{F}(x, \omega)+\frac{\omega^{2} \epsilon_{r}}{c^{2}} \tilde{F}(x, \omega)=0
$$

Consider now a one-dimensional resonator oriented along the $z$ axis with one boundary at $z=0$, one at $z=L$, and a traveling wave $E^{(i)}(\omega) e^{j k z}$ incident from the right (Fig. $1)$. In general, there will be a reflected wave $E^{(o)}(\omega)$ $e^{-j k z}$. The relation between the two can be written

$$
E^{(o)}(\omega)=R(\omega) E^{(i)}(\omega)
$$

for some function $R(\omega)$. Formally, this is equivalent to the boundary-value problem consisting of (3) and the boundary conditions

$$
\begin{aligned}
& {\left[\tilde{F}(z, \omega)-\left(\frac{1+r_{0}}{1-r_{0}}\right) \frac{1}{j k} \frac{\partial}{\partial z} \tilde{F}(z, \omega)\right]_{z=-L}=0} \\
& {\left[\tilde{F}(z, \omega)+\frac{1}{j k} \frac{\partial}{\partial z} \tilde{F}(z, \omega)\right]_{z=0}=2 E^{(i)}(\omega) .}
\end{aligned}
$$

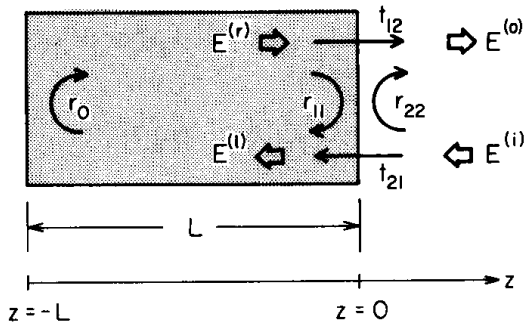

Fig. 1. Schematic of a laser cavity. The reflectivity of the left mirror is $r_{0}$, while the right mirror is characterized by scattering parameters $r_{11}, r_{22}$, $t_{12}$, and $t_{21}$. The length is $L$, and the complex index of refraction is $\mu$. The field amplitudes shown are the complex traveling-wave amplitudes.

$R(\omega)$ is given by

$$
\begin{aligned}
R(\omega)= & \frac{1}{2 E^{(i)}(\omega)} \\
& \cdot\left[\tilde{F}(z, \omega)-\frac{1}{j k} \frac{\partial}{\partial z} \tilde{F}(z, \omega)\right]_{z=0} .
\end{aligned}
$$

In general, $R$ will be complex and analytic. If we require that $R$ goes to infinity-that is, that we get an output field with no input - then we have a laser, and the roots to the equation $1 / R(\omega)=0$ define the lasing frequencies.

Derivation of the coupled-mode rate equations for a given resonator depends upon the analytic properties of the function $R(\omega)$. It is remarkably simple, however, if $R(\omega)$ satisfies several criteria.

1) The only singularities of $R(\omega)$ must be simple poles at a set of finite values denoted by $\left\{\omega_{n}\right\}$. Their residues are denoted by $\left\{\rho_{n}\right\}$ for $n=1,2, \cdots$.

2) The poles are numbered in order from an analytic point $\omega_{0}$ such that $0<\left|\omega_{1}-\omega_{0}\right|<\left|\omega_{2}-\omega_{0}\right|<\cdots$.

3) The function $R(\omega)$ is bounded for all $\omega \neq \omega_{n}$.

4) A set of closed contours $\left\{C_{n}\right\}$ can be chosen such that $C_{n}$ encloses the points $\omega_{1} \cdots \omega_{n}$; the minimum distance $R_{n}$ of $C_{n}$ from $\omega_{0}$ tends to infinity as $n \rightarrow \infty$, while the length $L_{n}$ of $C_{n}$ is $O\left(R_{n}\right)$.

If these conditions are satisfied, then the Mittag-Leffler theorem [8] allows us to write the function $R(\omega)$ as follows:

$$
R(\omega)=R\left(\omega_{0}\right)+\sum_{n=1}^{\infty}\left(\frac{\rho_{n}}{\omega-\omega_{n}}+\frac{\rho_{n}}{\omega_{n}-\omega_{0}}\right) .
$$

The sum in [7] converges uniformly for all $\omega \neq \omega_{n}$. Furthermore, if the series $\Sigma_{n=1}^{\infty} \rho_{n} /\left(\omega_{n}-\omega_{0}\right)$ converges, then we can write

$$
\begin{aligned}
R(\omega) & =\rho_{0}+\sum_{n=1}^{\infty} \frac{\rho_{n}}{\omega-\omega_{n}} \\
\rho_{0} & \equiv R\left(\omega_{0}\right)+\sum_{n=1}^{\infty} \frac{\rho_{n}}{\omega_{n}-\omega_{0}} .
\end{aligned}
$$

We define a set of variables $\left\{\tilde{E}_{n}^{(0)}\right\}$ such that

$$
\begin{aligned}
& \tilde{E}_{n}^{(o)} \equiv \frac{\rho_{n}}{\omega-\omega_{n}} \tilde{E}^{(i)} \quad \text { for } n=1,2, \cdots \\
& \tilde{E}_{0}^{(o)}=\rho_{0} \tilde{E}^{(i)}
\end{aligned}
$$


so that $\tilde{E}^{(o)}=\Sigma_{n=0}^{\infty} \tilde{E}_{n}^{(o)}$. A bit of algebraic manipulation yields the relation

$$
j \omega \tilde{E}_{n}^{(o)}=j \omega_{n} \tilde{E}_{n}^{(o)}+j \rho_{n} \tilde{E}^{(i)} .
$$

We now invert the Fourier transform to get the set of equations

$$
\begin{aligned}
E_{0}^{(o)}(t) & =\rho_{0} E^{(i)}(t) \\
\frac{\partial}{\partial t} E_{n}^{(o)}(t) & =j \omega_{n} E_{n}^{(o)}(t)+j \rho_{n} E^{(i)}(t) \\
& \text { for } n=1,2, \cdots .
\end{aligned}
$$

Equations (11a)-(11b) are a set of rate equations for field amplitudes in a laser cavity in the presence of an input field. Equation $(11 \mathrm{~b})$ resembles a single-mode rate equation with driving field, and it is therefore tempting to interpret $E_{n}^{(o)}(t)$ as the amplitude of the $n$th longitudinal (spatial) mode. While there is a spatial field distribution associated with each amplitude $E_{n}^{(o)}$, if $\tilde{E}^{(i)}(\omega) \neq 0$ for some $\omega \neq \omega_{n}$, the distribution is not generally that of a longitudinal mode which, by definition, is a solution to (3) with homogeneous boundary conditions.

This result should not be surprising. The modes of an isolated resonator do not form a complete set when an external field is present [11]. The fields $\tilde{F}_{n}(z)$ associated with the amplitudes $\tilde{E}_{n}^{(o)}(\omega)$ do, and therefore they are the "modes" we must use. The longitudinal modes may be taken as approximations to the true fields $\tilde{F}_{n}(z)$; an example is discussed in Appendix A.

Withal, the field is completely determined by the amplitudes $\tilde{E}_{n}^{(o)}$ that evolve according to (11a)-(11b). We may use these equations to analyze a coupled-cavity laser by letting the input $E^{(i)}$ of one laser be the output $E^{(o)}$ of the other and vice versa. Before doing that, we must calculate $R(\omega)$ and its analytic properties.

\section{A One-Dimensional Cavity}

We calculate the reflectivity function $R(\omega)$ for the cavity illustrated in Fig. 1. It is of length $L$ and is assumed to be filled with a gain medium of index $\mu$ that may be complex to accommodate gain and/or loss. One mirror has reflectivity $r_{0}$; the other, through which coupling takes place, is characterized by its reflection coefficients $r_{11}, r_{22}$ and transmission coefficients $t_{21}, t_{12}$. The traveling-wave amplitudes outside the cavity are given by $\tilde{E}^{(o)}$ and $\tilde{E}^{(i)}$; inside the cavity, we denote them by $\tilde{E}^{(r)}$ and $\tilde{E}^{(l)}$.

Inspection of Fig. 1 yields the following relations between the traveling-wave amplitudes:

$$
\begin{aligned}
& \tilde{E}^{(l)}=t_{21} \tilde{E}^{(i)}+r_{11} \tilde{E}^{(r)} \\
& \tilde{E}^{(r)}=r_{0} \exp (-2 j \beta L) \tilde{E}^{(l)} \\
& \tilde{E}^{(o)}=r_{22} \tilde{E}^{(i)}+t_{12} \tilde{E}^{(r)} .
\end{aligned}
$$

By eliminating $\tilde{E}^{(r)}$ and $\tilde{E}^{(l)}$ from $(12 \mathrm{a})-(12 \mathrm{c})$, we can find a linear relation between $\tilde{E}^{(o)}$ and $\tilde{E}^{(i)}$, and thus find $R(\omega)$.

$$
\begin{aligned}
R(\omega)= & r_{22}+r_{0} t_{12} t_{21} \\
& \cdot \frac{\exp (-2 j \omega \mu L / c)}{1-r_{0} r_{11} \exp (-2 j \omega \mu L / c)} .
\end{aligned}
$$

Clearly, $R$ satisfies the criteria for the Mittag-Leffler theorem. Its pole are found by setting the denominator of the fraction equal to zero and solving for $\omega$ :

$$
\begin{gathered}
r_{0} r_{11} \exp (-2 j \omega \mu L / c)=1 \\
\omega_{n}=\frac{c}{2 j \mu L}\left(\ln r_{0} r_{11}+2 j N \pi\right)
\end{gathered}
$$

for integral $N$. The residues may be determined by applying the following theorem.

If $R(\omega) \equiv f(\omega) / g(\omega)$ has a simple pole at $\omega=\omega_{n}$, and $\omega_{n}$ is an analytic point of $f(\omega)$, the residue $\rho_{n}$ is given by

$$
\rho_{n}=\frac{f\left(\omega_{n}\right)}{g^{\prime}\left(\omega_{n}\right)}
$$

Applying this to (13), we find

$$
\begin{aligned}
\rho_{n} & =\frac{r_{0} t_{12} t_{21} \exp \left(-2 j \omega_{n} \mu L / c\right)}{-r_{0} r_{11}\left(\frac{-2 j \mu L}{c}\right) \exp \left(-2 j \omega_{n} \mu L / c\right)} \\
= & \frac{c}{2 j \mu L} \frac{t_{12} t_{21}}{r_{11}} .
\end{aligned}
$$

Thus, all of the residues are equal.

We make a distinction here between the integer $n$ and the integer $N$. Condition (2) for the validity of the MittagLeffler theorem required that the poles be ordered in distance from the analytic point $\omega_{0}$, and that they be assigned the index $n=1,2,3 \cdots$. On the other hand, $N$ can take on values from $-\infty$ to $+\infty$. To preserve the ordering, we must order the values of $N$ in distance from a given value. An example will make this clear. If we begin at a point in the interval $N \in(0,1 / 2)$, then for $n=1,2,3,4,5$, $\cdots$, we take $N=0,1,-1,2,-2, \cdots$ to determine the order of the terms in each summation. This choice of ordering assures us that the summations in $(8 a)-(8 b)$ are conditionally convergent (they are not absolutely convergent).

Now let us evaluate the summation in (8b). We choose

$$
\omega_{0} \equiv \frac{c}{2 j \mu L}\left(\ln r_{0} r_{11}+j \pi\right)
$$

(which corresponds to the choice of $N$ in the example above). The summation becomes

$$
\begin{aligned}
& \sum_{n} \frac{t_{12} t_{21}}{j \pi r_{11}} \cdot \frac{1}{2 N-1}=\frac{t_{12} t_{21}}{j \pi r_{11}} \\
& \cdot\left\{\frac{1}{-1}+\frac{1}{1}+\frac{1}{-3}+\frac{1}{3}+\cdots\right\}=0 .
\end{aligned}
$$


Consequently,

$$
\rho_{0}=R\left(\omega_{0}\right)=r_{22}-\frac{1}{2} \frac{t_{12} t_{21}}{r_{11}} .
$$

\section{Two Axially Coupled Cavities}

To compose a set of coupled-mode equations for two coupled cavities, we must take the two-cavity system and split it into two "virtual" cavities, as illustrated in Fig. 2 . We label the two cavities by $a$ and $b$. Parameters of the two cavities will be labeled by a subscript; fields will be labeled by a superscript in parentheses. The coupling between the cavities will be characterized by transmission and reflection coefficients $t_{a b}, t_{b a}$ and $r_{a a}, r_{b b}$.

Our coupled-mode theory requires that in the absence of an input field, each cavity is an independent resonator with transmission and reflection coefficients $t_{12 a}, t_{21 a}, r_{11 a}$, $r_{22 a}$ for cavity $a$ and a similar set for cavity $b$. We partition the transmission and reflection coefficients of the coupled cavity between the two virtual cavities as follows:

$$
\begin{aligned}
t_{12 a} \equiv t_{a b}, t_{12 a} & \equiv 1 \\
r_{11 a} & \equiv r_{a a}, r_{22 a} \equiv 0
\end{aligned}
$$

with similar relations (with $a$ and $b$ reversed) for the other cavity. Using these definitions in (15) and (17), we find that

$$
\rho_{0 a}=-\frac{1}{2} \frac{t_{a b}}{r_{a a}}, \rho_{n a}=\frac{c}{2 j \mu_{a} L_{a}} \frac{t_{a b}}{r_{a a}} .
$$

If we use values in $(11 \mathrm{~b})$, with

$$
E_{a}^{(i)}=\sum_{n} E_{n}^{(b)}
$$

we get the familiar coupled-mode equations for coupledcavity lasers. However, there is an additional equation(11a)-that is not in the form of a rate equation, and that has not appeared in traditional coupled-mode treatments.

While $E_{n}^{(o)}$ is the amplitude of the $n$th longitudinal "mode" of one of the cavities, $E_{0}^{(o)}$ is nothing of the sort; it is the frequency-independent reflection of the incident field. Its effect is to change the apparent values of the coupling coefficients. We write (11a) for each of the two cavities:

$$
\begin{aligned}
& E_{0}^{(a)}=\rho_{0 a} \sum_{n=0}^{\infty} E_{n}^{(b)}=\rho_{0 a} E_{0}^{(b)}+\rho_{0 a} \sum_{n=1}^{\infty} E_{n}^{(b)} \\
& E_{0}^{(b)}=\rho_{0 b} E_{0}^{(a)}+\rho_{0 b} \sum_{n=1}^{\infty} E_{n}^{(a)} .
\end{aligned}
$$

We can eliminate $E_{0}^{(a)}$ and $E_{0}^{(b)}$ from the coupled-mode equations (11b) by using [from (23a)-(23b)]

$$
\begin{aligned}
E_{0}^{(b)}= & \frac{\rho_{0 b} \rho_{0 a}}{1-\rho_{0 b} \rho_{0 a}} \sum_{n=1}^{\infty} E_{n}^{(b)} \\
& +\frac{\rho_{0 b}}{1-\rho_{0 b} \rho_{0 a}} \sum_{n=1}^{\infty} E_{n}^{(a)}
\end{aligned}
$$

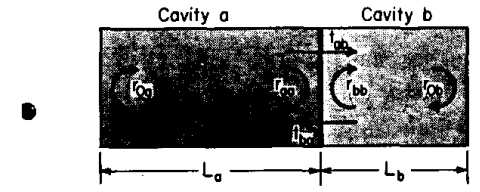

(a)

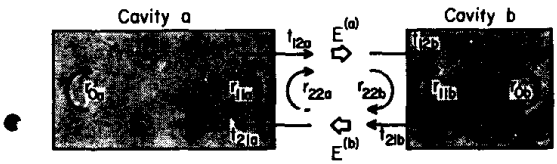

(b)

Fig. 2. Schematic of two coupled laser cavities. (a) The two-cavity system; the gap is characterized by scattering parameters $r_{a a}, r_{b b}, t_{a b}$, and $t_{b b}$. (b) The same system, split into two "virtual"' cavities.

and its companion relation with $a$ and $b$ reversed. Thus, if we define self coupling-coefficients $\kappa_{a a}, \kappa_{b b}$, and crosscoupling coefficients $\kappa_{a b}, \kappa_{b a}$, we can write a set of coupled-mode rate equations involving only the longitudinal modes. They are

$$
\begin{aligned}
\frac{\partial}{\partial t} E_{n}^{(a)} & =j \omega_{n a} E_{n}^{(a)}+\sum_{m=1}^{\infty} j\left(\kappa_{a a} E_{m}^{(a)}+\kappa_{a b} E_{m}^{(b)}\right) \\
\kappa_{a b} & =\frac{\rho_{n a}}{1-\rho_{0 a} \rho_{0 b}}, \quad \kappa_{a a}=\rho_{0 b} .
\end{aligned}
$$

The equations for cavity $b$ are the same with reversed subscripts.

Now we substitute the values for $\rho_{i j}$ from (22). We define a "coupling factor" $K$ as

$$
K \equiv-\frac{t_{a b} t_{b a}}{r_{a a} r_{b b}}
$$

$K$ may be complex. Then

$$
\begin{aligned}
\kappa_{a b} & =\frac{c}{2 j \mu_{a} L_{a}} \cdot \frac{t_{a b} / r_{a a}}{1+K / 4}, \\
\kappa_{a a} & =\frac{c}{2 j \mu_{a} L_{a}} \cdot \frac{K / 2}{1+K / 4} .
\end{aligned}
$$

For a lossless coupling, $K$ is real and positive. For lossy couplings-for example, the coupling provided by an air gap of a small number of half wavelengths $-K$ can become purely negative and of magnitude greater than or equal to 4 [9], [10]

If $K$ approaches -4 , the coupling coefficients diverge. This phenomenon has no physical basis; such a laser will remain well behaved. As we will show in Appendix B, the divergence occurs because the individual cavity modes become overcomplete as a description of the coupled system, in which case there is no exact, nonsingular representation of the coupled system in individual cavity modes.

The value of the cross-coupling coefficients will vary with chosen field normalization. For example, if we define $E_{n}^{(a)}$ as the traveling-wave amplitude (as we have done here), the cross-coupling coefficients will be different from what we would find if we defined $\left|E_{n}^{(a)}\right|^{2}$ as the total en- 
ergy in the field (as was done in [5] and [6]). Since the field normalization depends on the partitioning of the transmission coefficients in (21), that, too, can change the cross-coupling coefficients. However, the self-coupling coefficients and the product of the cross-coupling coefficients are independent of field normalization. In the comparisons that follow, all of the coefficients have been adjusted to have the same field normalization.

In Fig. 3, for a system of two identical cavities with a lossless coupling, we plot $\left|\kappa_{a b}\right|$ as derived from heuristic (5), overlap integral (6), local field (11), and the present treatments. The first three treatments give the same values; they are close to the exact results for $\left|t_{12}\right| \leq 0.8$. For a highly lossy gap $\left(t_{a b}, t_{b a} \rightarrow 0\right.$ as $r_{a a}, r_{b b}$ remain fixed), the local field and exact coefficients both approach the same value:

$$
\kappa_{a b} \rightarrow \frac{c}{2 j \mu_{a} L_{a}} \cdot \frac{t_{a b}}{r_{a a}} .
$$

This result is also in agreement with the reported results from the overlap-integral treatment. (The heuristic formulas were derived under the explicit assumption that the coupling was lossless; they should not be applied to the case of a lossy coupling.) In Fig. 4, we plot the magnitudes of the self-coupling coefficients from local field theory along with the exact results.

The figures illustrate that the coefficients derived from all four approaches are generally within a factor of 2 or so of each other. Considering that other approximations in the analysis-the adiabatic approximation, for example, or truncation of the set of modes-may take an equal or greater toll on quantitative accuracy, it may not make much difference which formulas are used. However, since this derivation is simple, analytical-and exact-we think it is preferable. The method can be generalized to higher dimensional problems.

\section{APPENDIX A}

In the spirit of coupled-mode theory, we would like to expand the field inside the resonator in terms of the modal amplitudes as

$$
\tilde{F}(z, \omega)=\sum_{n} \tilde{F}_{n}(z) \tilde{E}_{n}^{(o)}(\omega)
$$

We will perform this expansion for the laser of Fig. 1 for the special case $r_{22}=0$. From $(12 a)-(12 c)$, we find the traveling-wave amplitudes

$$
\begin{aligned}
& \tilde{E}^{(r)}=\frac{\tilde{E}^{(o)}}{t_{12}} \\
& \tilde{E}^{(l)}=\frac{e^{2 j \beta L}}{r_{0} t_{12}} \tilde{E}^{(o)}
\end{aligned}
$$

where $\beta \equiv \omega \mu / c$. By inspection of Fig. 1, the field inside

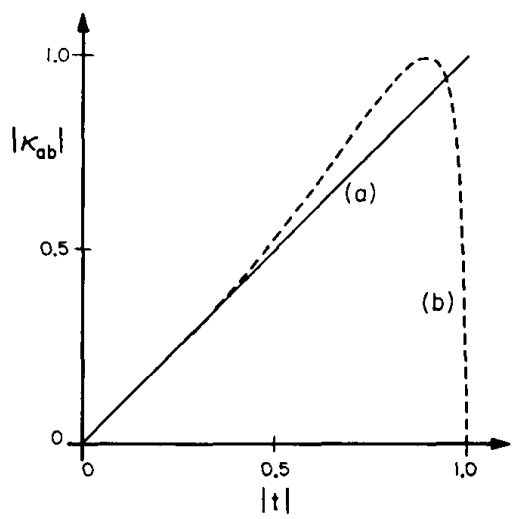

Fig. 3. Plot of $\left|\kappa_{a b}\right|$ versus transmission for the different treatments, normalized to $c / 2 j \mu L$. (a) Heuristic, overlap integral or local field, (b) exact.

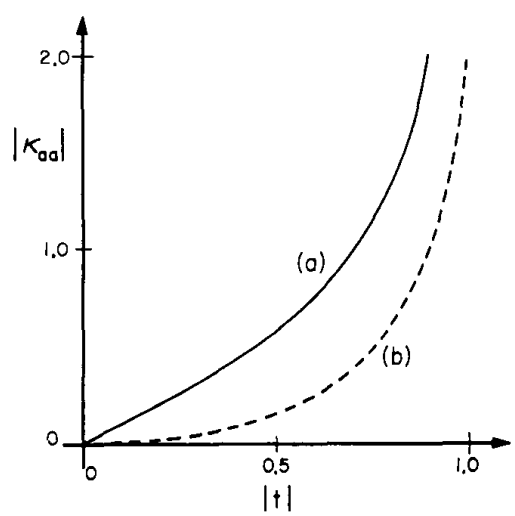

Fig. 4. Plot of $\left|\kappa_{a a}\right|$ versus transmission for (a) local field, (b) exact.

the laser must be

$$
\begin{aligned}
\tilde{F}(z, \omega) & =\tilde{E}^{(r)} e^{-j \beta z}+\tilde{E}^{(l)} e^{+j \beta z} \\
& =\frac{1}{r_{0} t_{12}}\left(r_{0} e^{-j \beta z}+e^{j \beta(z+2 L)} \mathrm{E}^{(0)} .\right.
\end{aligned}
$$

Comparison of (A1), (A4), and the definition of $E_{n}^{(o)}$ shows that if a representation such as (A1) exists, it must have

$$
\tilde{F}_{n}(z)=\frac{1}{r_{0} t_{12}}\left(r_{0} e^{-j \beta z}+e^{j \beta(z+2 L)}\right) .
$$

Equation (A5) is less than ideal. Not only do all the $\tilde{F}_{n}(z)$ 's have the same form, they are explicit functions of $\omega$, and therefore are not stationary in time.

However, in the absence of an input field, $\tilde{E}_{n}^{(o)}$ takes the form $A_{n} \delta\left(\omega-\omega_{n}\right)$ for a constant $A_{n}$. The product $\tilde{E}_{n}^{(o)}(\omega) \tilde{F}_{n}(z ; \omega)$ may thereby be written $A_{n} \delta\left(\omega-\omega_{n}\right)$ $\tilde{F}_{n}\left(z ; \omega_{n}\right)$ with no loss of accuracy. Only this case is $\tilde{F}_{n}(z)$ independent of $\omega$ and stationary in time; and of course, it is then the $n$th longitudinal mode of the isolated resonator. 


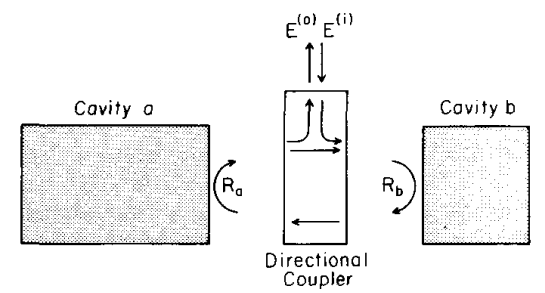

Fig. 5. Coupled-cavity system with a fictitious directional coupler in the gap. All transmission coefficients are unity for plane waves traveling in the direction of the arrows.

\section{APPENDIX B}

As $K$ approaches -4 , or equivalently, $\rho_{0 a} \rho_{0 b}$ approaches 1 , the coupling coefficients diverge. To analyze this situation, we must examine the coupled structure within a composite-cavity representation.

To do this systematically, we introduce a fictitious directional coupler into the system of virtual cavities, as illustrated in Fig. 5. The coupler passes light from one laser to the other unchanged, but it allows us to inject a signal $E^{(i)}$ and extract a probe $E^{(o)}$, permitting application of the formalism of Section II.

We find a relation between $E^{(i)}$ and $E^{(o)}$ in the same way we found (13). If the reflectivities of the two cavities are $R_{a}(\omega)$ and $R_{b}(\omega)$, then the reflectivity seen at the directional coupler is

$$
R_{c}(\omega)=\frac{E^{(o)}}{E^{(i)}}=\frac{R_{a}(\omega) R_{b}(\omega)}{1-R_{a}(\omega) R_{b}(\omega)} .
$$

Each pole of $R_{c}(\omega)$ determines a mode of the composite cavity, just as the poles of $R_{a}$ and $R_{b}$ determine the modes of the individual cavities.

Now consider the situation when $R_{a}$ and $R_{b}$ each possess a finite number of poles and $\rho_{0 a} \rho_{0 b} \rightarrow 1$. In accordance with $(8 \mathrm{a})$, we write

$$
R_{a}(\omega)=\rho_{0 a}+\sum_{n=1}^{N_{a}} \frac{\rho_{n a}}{\omega-\omega_{n a}}
$$

and a similar equation for $R_{b}$. We can write this as a single ratio of two polynomials:

$$
R_{a}(\omega)=\frac{\rho_{0 a} \omega^{N_{a}}+\cdots}{\prod_{n=1}^{N_{a}}\left(\omega-\omega_{n a}\right)}
$$

where $(\cdots)$ indicates lower powers of $\omega$. Then
They are spanned by the union of the $N_{a}$ modes of cavity $a$ with the $N_{b}$ modes of cavity $b$. However, if $\rho_{0 a} \rho_{0 b} \rightarrow$ 1 , the denominator becomes only of order $\left(N_{a}+N_{b}-\right.$ 1). We find ourselves trying to uniquely represent an $\left(N_{a}\right.$ $\left.+N_{b}-1\right)$-dimensional space with $\left(N_{a}+N_{b}\right)$ vectors. Clearly, the set of individual cavity modes is overcomplete. Just as clearly, this situation will persist no matter how many poles lie in the spectrum of $R_{a}$ and $R_{b}$. Therefore, there is no exact solution for $K=-4$. In this situation, an approximate theory (e.g., [5], [11]) will give more meaningful results.

\section{REFERENCES}

[1] W. T. Tsang, N. A. Olsson, and R. A. Logan, "High-speed direct single-frequency modulation with large tuning rate and frequency excursion in cleaved-coupled-cavity semiconductor lasers," Appl. Phys. Lett., vol. 42, pp. 650-652, 1983.

[2] L. A. Coldren, G. D. Boyd, J. E. Bowers, and C. A. Burrus, "Reduced dynamic linewidth in three-terminal two-section diode lasers," Appl. Phys. Lett., vol. 46, pp. 125-127, 1985.

[3] R. J. Lang and A. Yariv, "Semiclassical theory of noise in multielement semiconductor lasers," IEEE J. Quantum Electron., vol. QE22, pp. 436-449, Mar. 1986.

[4] M. B. Spencer and W. E. Lamb Jr., "Theory of two coupled lasers," Phys. Rev. A, vol. 5, pp. 893-898, 1972.

[5] D. Marcuse, "Coupled-mode theory of optical resonant cavities," IEEE J. Quantum Electron., vol. QE-21, pp. 1819-1826, Nov. 1985.

[6] - "Coupling coefficients of coupled laser cavities," IEEE J. Quantum Electron., vol. QE-22, pp. 223-226, Feb. 1986.

[7] R. J. Lang, Ph.D. dissertation, California Inst. Technol., Pasadena, 1986.

[8] E. C. Titchmarsh, The Theory of Functions. London: Oxford, 1932, p. 110.

[9] L. A. Coldren and T. L. Koch, "Analysis and design of coupledcavity lasers, Part I: Threshold gain analysis and design guidelines,' IEEE J. Quantum Electron., vol. QE-20, pp. 659-670, June 1984.

[10] C. H. Henry and R. F. Kazarinov, "Stabilization of single-frequency operation of coupled cavity lasers," IEEE J. Quantum Electron., vol. QE-20, pp. 733-744, July 1984.

$$
\begin{aligned}
R_{c} & =\frac{\left(\rho_{0 a} \omega^{N_{a}}+\cdots\right)\left(\rho_{0 b} \omega^{N_{b}}+\cdots\right)}{\prod_{n=1}^{N_{a}}\left(\omega-\omega_{n a}\right) \prod_{m=1}^{N_{b}}\left(\omega-\omega_{m b}\right)-\left(\rho_{0 a} \omega^{N_{a}}+\cdots\right)\left(\rho_{0 b} \omega^{N_{b}}+\cdots\right)} \\
& =\frac{\left(\rho_{0 a} \rho_{0 b} \omega^{N_{a}+N_{b}}+\cdots\right)}{\left(\left(1-\rho_{0 a} \rho_{0 b}\right) \omega^{N_{a}+N_{b}}+\cdots\right)} .
\end{aligned}
$$

The poles of $R_{c}(\omega)$ determine the lasing modes; since the denominator of (B5) is an $\left(N_{a}+N_{b}\right)$-order polynomial, there are $\left(N_{a}+N_{b}\right)$ modes of the coupled system.
[11] R. J. Lang and A. Yariv, "Local field theory for coupled optical resonators," Phys. Rev. A, vol. 34, pp. 2038-2043, 1986. 
Robert J. Lang (S'83-M'86) was born in Dayton, Ohio, on May, 4, 1961, and was raised in Atlanta, Georgia. In 1982, he received the B.S. degree in electrical engineering from the California Institute of Technology, Pasadena. He received the M.S. degree, also in electrical engineering, from Stanford University, Stanford, CA, in 1983. In 1986, he received the Ph.D. degree in applied physics from Caltech, for work on coupled-cavity and unstable resonator semiconductor lasers.

In 1987 he was a guest scientist at the Standard Elektrik Lorenz Research Center in Stuttgart, West Germany. In 1988 he joined the Jet Propulsion Laboratory at the California Institute of Technology, where he is studying dynamic and spectral properties of semiconductor lasers. He is the author or coauthor of over twenty technical papers.

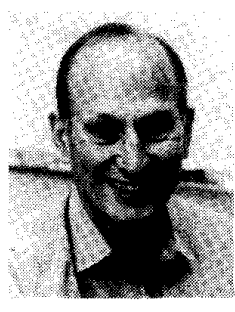

Amnon Yariv (S'56-M'59-F'70) was born in Tel Aviv, Israel, on April 13, 1930. He received the B.S., M.S., and Ph.D. degrees in electrical engineering from the University of California, Berkeley, in 1954, 1956, and 1958, respectively.

A veteran of the Israeli war of independence, he came to the United States in 1951. He went to Bell Laboratories, Murray Hill, NJ, in 1959, joining the California Institute of Technology, Pasadena, in 1964, as an Associate Professor of Electrical Engineering, becoming a Professor in 1966.
In 1980 he became the Thomas G. Myers Professor of Electrical Engineering and Applied Physics. On the technical side, he took part (with various co-workers) in the discovery of a number of early solid-state laser systems, in the formulation of the theory of parametric quantum noise and the prediction of parametric fluorescence, in the invention of the technique of mode-locked ultrashort-pulse lasers and FM lasers, in the introduction of GaAs and $\mathrm{CdTe}$ as infrared electrooptic and window materials, in proposing and demonstrating semiconductor-based integrated optics technology, and in pioneering the field of phase conjugate optics. His present research efforts are in the areas of nonlinear optics, recombination mechanisms in semiconductors, semiconductor lasers and integrated optics, especially the problem of monolithic integration of transistors, injection lasers, and detectors for high-frequency applications. He has authored or coauthored some 300 papers in professional journals, as well as a number of basic texts in quantum electronics, optics, and quantum mechanics. He is a Consultant to the Hughes Aircraft Research Laboratories, and is a founder and chairman of the Board of Ortel Corporation, Alhambra, CA.

Dr. Yariv is a member of the American Physical Society, Phi Beta Kappa, and the National Academy of Engineering, and a Fellow of the Optical Society of America. He was the recipient of the 1980 Quantum Electronics Award of the IEEE. He is also an Associate Editor of Optics Communications and the Journal of Applied Physics, and was previously an Associate Editor of the IEEE JOURNAL OF QUANTUM EleCtronics. 